\title{
Searching for factors associated with resistance to acetylsalicylic acid used for secondary prevention of stroke
}

\author{
Beata Łabuz-Roszak ${ }^{1}$, Krystyna Pierzchała ${ }^{1}$, Ewa Niewiadomska², Michał Skrzypek², \\ Agnieszka Machowska-Majchrzak ${ }^{1}$
}

\author{
${ }^{1}$ Chair and Clinical Department of Neurology in Zabrze, Medical University of Silesia, \\ Katowice, Poland \\ ${ }^{2}$ Department of Biostatistics, Faculty of Public Health, Medical University of Silesia, \\ Katowice, Poland
}

Submitted: 19 December 2012

Accepted: 1 March 2013

Arch Med Sci 2015; 11, 1: 106-114

DOI: 10.5114/aoms.2015.49211

Copyright (C) 2015 Termedia \& Banach

\section{Abstract}

Introduction: The aim of the study was to evaluate the prevalence of resistance to acetylsalicylic acid (ASA), used for secondary prevention of stroke, including the assessment of risk factors associated with the lack of ASA anti-aggregatory action.

Material and methods: Patients after a transient ischaemic attack (TIA) or ischaemic stroke in the acute $(n=111)$ and chronic phase $(n=87)$ were enrolled in the study. The assessment of platelet function was performed by whole blood impedance aggregometry using a multi-channel platelet function analyser (Multiplate).

Results: A proper response to ASA was found in 121 patients (61.1\%) (ASA responders), a partial response to ASA in 59 patients (29.8\%) (ASA partial responders), and ASA resistance in 18 patients (9.1\%) (ASA non-responders). Acetylsalicylic acid resistance was observed more frequently in the chronic phase. The mean low-density lipoprotein (LDL) concentration was higher in ASA non-responders $(p=0.02)$. The mean heart rate $(p=0.03)$ and the mean haematocrit $(p=0.03)$ were higher in the group of ASA partial responders and ASA non-responders. Angiotensin II receptor antagonists were more often used in the group of ASA partial responders and ASA non-responders $(p=0.04)$. Diuretics were more rarely used by ASA non-responders, whereas fibrates were more rarely used by ASA partial responders.

Conclusions: The method enabled the detection of ASA resistance in some patients with cerebrovascular disease. The study revealed some possible risk factors of ASA resistance: long ASA therapy, increased heart rate, higher LDL concentration, and higher haematocrit value. The relationship between the effect of ASA and other medications (angiotensin II receptor blockers, fibrates, diuretics) requires further study. Platelet function monitoring should be considered in patients at a greater risk of ASA resistance.

Key words: acetylsalicylic acid, stroke, resistance to acetylsalicylic acid, impedance aggregometry.

\section{Introduction}

Acetylsalicylic acid (ASA) is the major drug used for ischaemic stroke prevention. It was synthesised by Felix Hoffman in 1897 and was introduced for treatment in 1899 under the name "aspirin", as it is commonly

\author{
Corresponding author: \\ Beata Łabuz-Roszak MD, PhD \\ Department of Neurology \\ Medical University of Silesia \\ 13/15 3 Maja St \\ 41-800 Zabrze, Poland \\ Phone: +48 323704594 \\ E-mail: \\ beatamaria.pl@hoga.pl
}


known nowadays. In the 1960 s scientists discovered the antiplatelet properties of ASA $[1,2]$. In patients who regularly take ASA a $25 \%$ reduction of the incidence of myocardial infarction and ischaemic stroke was reported [2].

Despite the proven benefits of ASA which are connected with the significant decrease in cardiovascular events in those who regularly take this medication, some patients show what is termed "ASA resistance". This term is used when a new cardiovascular episode is noted in individuals who regularly take ASA (known as clinical resistance). The term "ASA resistance" is also used when laboratory test results indicate the lack of anti-aggregatory ASA action (known as laboratory resistance).

As most studies and meta-analyses show, there is a correlation between laboratory resistance and clinical resistance, i.e. more frequent incidence of cardiovascular events in patients with proven ASA resistance in laboratory tests [3-6].

The mechanism of ASA resistance is not fully understood as yet. In the available literature genetic factors are considered together with patient non-compliance, an inappropriate form or dose of the medication, and interactions with other agents and substances [7].

The aim of the study was to evaluate the prevalence of resistance to ASA used for secondary prevention of stroke including the assessment of risk factors associated with the lack of ASA antiaggregatory action.

\section{Material and methods}

Patients with a diagnosed transient ischaemic attack (TIA) or ischaemic stroke (both acute and chronic) who were administered ASA at the dose of 75-150 mg/day were enrolled in the study. The study was conducted between 1 February 2010 and 30 June 2011. Patients were treated at the Department of Neurology or Out-patient Neurological Clinic.

The inclusion criteria were the following: history of cerebral ischaemic episode (TIA or ischaemic stroke), regular ASA intake at the dose of 75$150 \mathrm{mg} /$ day from the time of onset of the disease, not using other antiplatelet and/or antithrombotic agents, and the patient's informed written consent for the study.

The exclusion criteria were the following: the intake of other antiplatelet agents, the use of other non-steroidal anti-inflammatory drugs (NSAIDs), irregular ASA intake, the use of heparin or low-molecular weight heparin, platelet count $<150 \times 10^{3} / \mu$ l or $>450 \times 10^{3} / \mu$ l, history of haemorrhage, haemoglobin concentration $<10 \mathrm{mg} / \mathrm{dl}$, lack of patient's informed written consent. The study was approved by the local Bioethics Committee.
All patients underwent physical and neurological examination. A $10 \mathrm{ml}$ fasting blood sample was obtained to determine platelet function and perform additional laboratory tests (CBC, glucose, total cholesterol, LDL, $\mathrm{HDL}$, triglycerides, $\mathrm{HbA}_{1 \mathrm{c}}$, CRP, creatinine, AspAT, AIAT, bilirubin, INR, APTT). The neurological status of patients was based on the National Institutes of Health Stroke Scale (NIHSS) and the modified Rankin scale (mRankin). The type of stoke was established according to the TOAST classification.

The platelet function test was performed by the method of whole blood impedance aggregometry using the multiple platelet function analyser Multiplate (Dynabyte) [8-10]. In all the study patients the ASPI test was performed with arachidonic acid as the platelet activator. The study was conducted in accordance with the following protocol: blood $(2.6 \mathrm{ml})$ was collected from each patient into the Sarstedt r-hirudin-type tube. The analysis was performed after at least 30 min, but not more than $2 \mathrm{~h}$ after blood sampling. Next, the assessment was performed using the Multiplate analyser. Initially $300 \mu \mathrm{l}$ of sodium chloride heated to the temperature of $37^{\circ} \mathrm{C}$ and $300 \mu \mathrm{l}$ of whole blood were placed in a special single-use testing chamber with a magnetic stirrer, previously placed in a proper measurement site and plugged into the analyser. Next, after 3 min of incubation, $20 \mu \mathrm{l}$ of arachidonic acid was added. Aggregation results were obtained after the following $6 \mathrm{~min}$, as the mean of two measurements in the form of a curve on the basis of which the area under the curve (AUC) was determined. Figure 1 presents an example of platelet function analysis (ASPI test) using the multiple platelet function analyser (Multiplate) in an ASA non-responder (resistance to ASA was recognized) and in an ASA responder (proper response to ASA).

The AUC reference range for the ASPI test was 691-1294 [AU*min] in accordance with the manufacturer's recommendations. These values were obtained at the Clinic of Homeostasis and Transfusion Medicine of the University of Munich by measuring platelet function using the Multiplate analyser in 66 healthy volunteers who were not taking any medications [10].

Based on the literature data, it was accepted that in patients who take ASA the value of AUC $<300$ indicates aspirin sensitivity (ASA responders), the value of AUC 300-690 indicates partial sensitivity (ASA partial responders), and the value of AUC > 690 indicates aspirin resistance (ASA non-responders) [10].

\section{Statistical analysis}

The obtained results underwent statistical analysis using the $\mathrm{R}$ software package 2.11.1, 

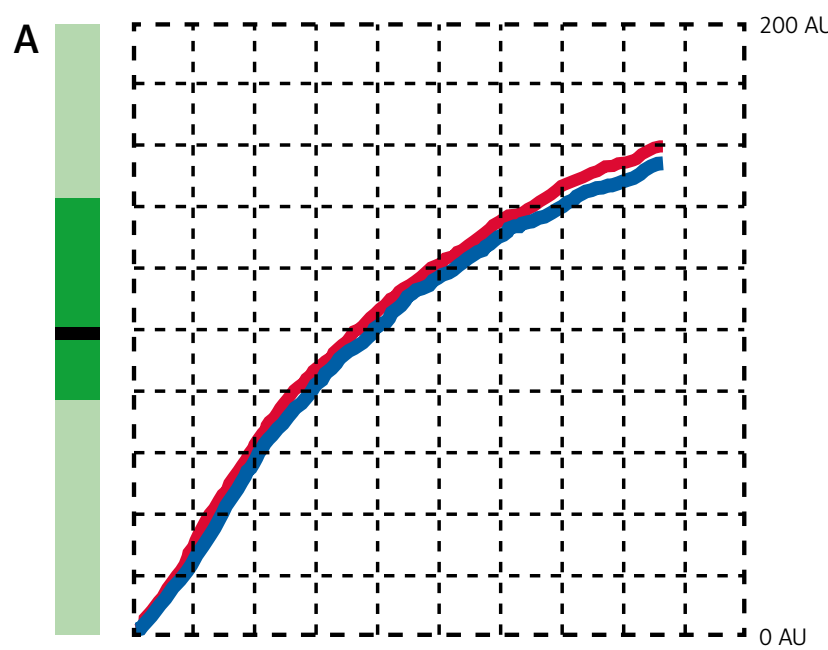

ASPI test

Area under curve: $896 \mathrm{AU} \times \min (691-1296)$

Aggregation: RUO: $157.1 \mathrm{AU}$

Velocity: RUO: $19.5 \mathrm{AU} / \mathrm{min}$

B

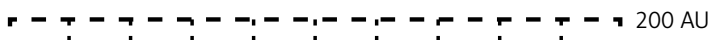

ASPI test

Area under curve: $262 \mathrm{AU} \times \min (691-1294)$

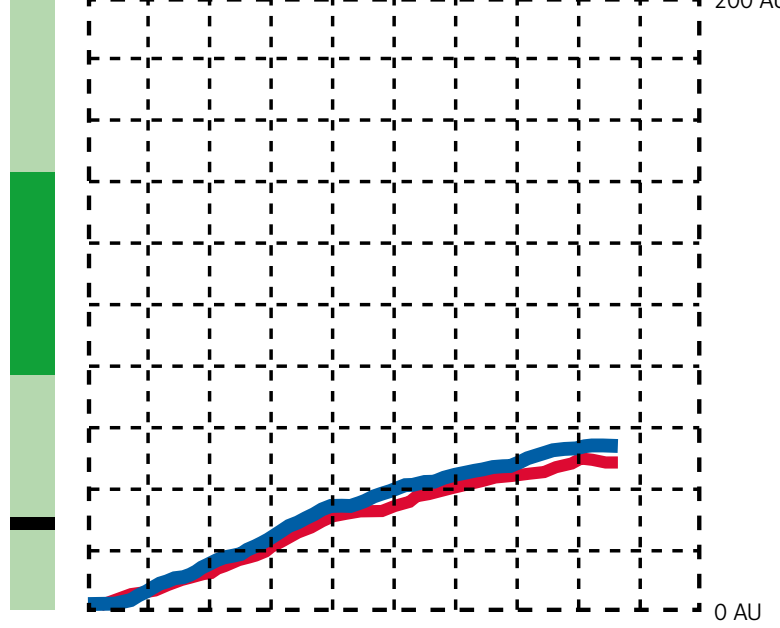

Aggregation: RUO: $50.3 \mathrm{AU}$

Velocity: RUO: $6.5 \mathrm{AU} / \mathrm{min}$

Figure 1. An example of platelet function analysis (ASPI test) using the multiple platelet function analyser (Multiplate): A - in ASA non-responder (resistance to acetylsalicylic acid), B - in ASA responder (proper response to acetylsalicylic acid)

R Foundation for Statistical Computing and Statistica v.9.0, StatSoft Poland. In data description the standard statistical parameters were provided, i.e. the number $N$, arithmetic mean $X$, the standard deviation (SD) and percentages (\%). Normal distribution of data was assessed by the Shapiro-Wilk normality test. Statistical significance of between-group differences was verified by Student's t-test or ANOVA, with the post-hoc least significant difference test, for normally distributed variables; otherwise the non-parametric Mann-Whitney $U$ test or Kruskal-Wallis test was used. The $\chi^{2}$ test, Yates' $\chi^{2}$ test or Fisher's exact test with Bonferroni method for pairwise comparisons of proportions was used for comparison of qualified variables. Statistical significance was based on the $p<0.05$ criterion.

\section{Results}

One hundred and ninety-eight patients were enrolled in the study including 111 patients hos- pitalised at the Department of Neurology (history of TIA or the acute phase of ischaemic stroke, $<1$ month from the onset of the disease) and 87 patients treated in the Out-patient Neurological Clinic (history of TIA or the chronic phase of ischaemic stroke $>1$ month from the onset of the disease). The clinical characteristics of the study patients are shown in Tables I, II.

The study patients were divided into three groups depending on the response to ASA, i.e. patients sensitive to ASA action (ASA responders, $R$ ) (AUC < 300), patients partially sensitive to ASA action (ASA partial responders, PR) (AUC $\geq 300$ and $\leq 690$ ), patients resistant to ASA action (ASA non-responders, NR) (AUC > 690). Additionally, a fourth group consisting of ASA partial responders and ASA non-responders (PR + NR) (AUC $\geq 300)$ was created for statistical purposes.

The incidence of the particular response types to ASA activity is shown in Table III. Acetylsalicylic acid resistance was found more frequently in patients with the chronic phase of ischaemic stroke. 
Table I. Clinical characteristics of examined patients

\begin{tabular}{|lccc|}
\hline Variable & $\begin{array}{c}\text { All patients } \\
(N=198)\end{array}$ & $\begin{array}{c}\text { Patients in the acute } \\
\text { phase } \\
(N=111)\end{array}$ & $\begin{array}{c}\text { Patients in the } \\
\text { chronic phase } \\
(\boldsymbol{N}=87)\end{array}$ \\
\hline Female/male, $n(\%)$ & $92(46.5) / 106(53.5)$ & $57(51.4) / 54(48.6)$ & $35(40.2) / 52(59.8)$ \\
\hline TIA/stroke, $n(\%)$ & $33(16.7) / 165(83.3)$ & $10(9) / 101(91)$ & $23(26.4) / 64(73.6)$ \\
\hline ASA 75 mg/ASA 150 mg, $n(\%)$ & $36(18.2) / 162(81.8)$ & $9(8.1) / 102(91.9)$ & $27(31) / 60(69)$ \\
\hline Age, mean \pm SD [years] & $67 \pm 12$ & $70 \pm 12$ & $64 \pm 11$ \\
\hline $\begin{array}{l}\text { Time from onset of TIA/stroke, } \\
\text { mean } \pm \text { SD [days] }\end{array}$ & $76.5 \pm 14.8$ & $6 \pm 4$ & $4.3 \pm 4$ \\
\hline Body weight, mean \pm SD [kg] & $27.6 \pm 4.9$ & $27 \pm 5.4$ & $78.4 \pm 13.7$ \\
\hline BMI, mean \pm SD [kg/m²] & $74.7 \pm 6.5$ & $75.4 \pm 6.3$ & $28.1 \pm 4.4$ \\
\hline Heart rate, mean \pm SD [bpm] & $134.3 \pm 11.3$ & $133.6 \pm 11.9$ & $13.8 \pm 6.7$ \\
\hline Systolic blood pressure, mean \pm SD [mm Hg] & $77.3 \pm 6.8$ & $77.1 \pm 7.1$ & $77.4 \pm 6.5$ \\
\hline Diastolic blood pressure, mean \pm SD [mm Hg] & $4.8 \pm 4$ & $7.3 \pm 6.9$ & $1.6 \pm 1.6$ \\
\hline NIHSS, mean \pm SD [points] & $1.8 \pm 1.5$ & $2.6 \pm 1.5$ & $0.9 \pm 0.8$ \\
\hline mRankin mean \pm SD [points] & & & \\
\hline
\end{tabular}

ASA - acetylsalicylic acid, TIA - transient ischaemic attack, BMI - body mass index, NIHSS - National Institutes of Health Stroke Scale, $S D$ - standard deviation.

Clinical characteristics of the various ASA response groups are shown in Table IV. The occurrence of risk factors of cardiovascular diseases, the intake of medications and the results of laboratory tests are shown in Tables V-VII. Since there was a small number of ASA non-responders, the statistical analysis concerning the relationship be- tween various factors and the occurrence of ASA resistance was performed for the whole study patient population (acute and chronic phases altogether).

No relationship was found between platelet aggregation and gender, age, type of stroke, dose of ASA, the result in NIHSS and mRankin, weight,

Table II. Stroke type according to TOAST classification

\begin{tabular}{|lccc|}
\hline Parameter & $\begin{array}{c}\text { All patients } \\
(N=165) \\
n(\%)\end{array}$ & $\begin{array}{c}\text { Patients in the acute phase } \\
\text { of stroke }(\boldsymbol{N}=101) \\
n(\%)\end{array}$ & $\begin{array}{c}\text { Patients in the chronic phase } \\
\text { of stroke }(\boldsymbol{N}=64) \\
n(\%)\end{array}$ \\
\hline LAA & $46(27.9)$ & $36(35.7)$ & $10(15.6)$ \\
\hline SAO & $87(52.7)$ & $44(43.6)$ & $43(67.2)$ \\
\hline CE & $18(10.9)$ & $15(14.8)$ & $3(4.7)$ \\
\hline UND & $14(8.5)$ & $6(5.9)$ & $8(12.5)$ \\
\hline
\end{tabular}

LAA - large-artery atherosclerosis, SAO - small-artery occlusion, CE - cardioembolism, UND - stroke of undetermined cause.

Table III. Response to ASA in the examined patients

\begin{tabular}{|c|c|c|c|c|}
\hline Variable & $\begin{array}{l}\text { All patients } \\
(N=198) \\
n(\%)\end{array}$ & $\begin{array}{c}\text { Patients in the acute } \\
\text { phase (I) }(N=111) \\
n(\%)\end{array}$ & $\begin{array}{c}\text { Patients in the chronic } \\
\text { phase (II) }(N=87) \\
n(\%)\end{array}$ & $\begin{array}{l}\text { Value of } p^{*} \\
\text { I vs. II }\end{array}$ \\
\hline ASA responders (R) & $121(61.1)$ & $74(66.7)$ & $47(54)$ & $\begin{array}{l}0.07 \\
\text { NS }\end{array}$ \\
\hline ASA partial responders (PR) & $59(29.8)$ & $30(27)$ & $29(33.3)$ & $\begin{array}{l}0.34 \\
\text { NS }\end{array}$ \\
\hline ASA non-responders (NR) & $18(9.1)$ & $7(6.3)$ & $11(12.7)$ & $\begin{array}{l}0.12 \\
\text { NS }\end{array}$ \\
\hline $\begin{array}{l}\text { ASA partial responders and } \\
\text { non-responders }(P R+N R)\end{array}$ & $77(38.9)$ & $37(33.3)$ & $40(46)$ & $\begin{array}{l}0.07 \\
\text { NS }\end{array}$ \\
\hline
\end{tabular}

ASA - acetylsalicylic acid. *Bonferroni pairwise comparisons for proportions. 
Table IV. Clinical characteristics of patients in various ASA response groups

\begin{tabular}{|c|c|c|c|c|c|c|}
\hline Variable & $\begin{array}{c}\text { ASA re- } \\
\text { sponders } \\
(\mathrm{R}) \\
(N=121)\end{array}$ & $\begin{array}{c}\text { ASA partial } \\
\text { responders } \\
(P R) \\
(N=59)\end{array}$ & $\begin{array}{l}\text { ASA non-re- } \\
\text { sponders } \\
(\mathrm{NR}) \\
(N=18)\end{array}$ & $\begin{array}{l}\text { ASA partial } \\
\text { responders } \\
\text { and non-re- } \\
\text { sponders } \\
(P R+N R) \\
(N=77)\end{array}$ & $\begin{array}{l}\text { Value of } p^{*} \\
\text { R vs. NR }\end{array}$ & $\begin{array}{l}\text { Value } \\
\text { of } p^{\star *} \\
\text { R vs. } \\
\text { PR + NR }\end{array}$ \\
\hline Female, $n(\%)$ & $55(45.5)$ & $26(44.1)$ & $11(61.1)$ & $37(48)$ & \multirow{2}{*}{$\begin{array}{l}0.2 \\
\text { NS }\end{array}$} & \multirow{2}{*}{$\begin{array}{l}0.7 \\
\text { NS }\end{array}$} \\
\hline Male, $n(\%)$ & $66(54.5)$ & $33(55.9)$ & $7(38.9)$ & $40(51.9)$ & & \\
\hline TIA, $n(\%)$ & $23(19)$ & $9(15.3)$ & $1(5.6)$ & $10(13)$ & \multirow{2}{*}{$\begin{array}{l}0.3 \\
\text { NS }\end{array}$} & \multirow{2}{*}{$\begin{array}{l}0.27 \\
\text { NS }\end{array}$} \\
\hline Stroke, $n(\%)$ & $98(81)$ & $50(84.7)$ & $17(94.4)$ & $67(87)$ & & \\
\hline ASA [75 mg], $n(\%)$ & $19(15.7)$ & $12(20.3)$ & $6(33.3)$ & $18(23.4)$ & \multirow{2}{*}{$\begin{array}{l}0.2 \\
\text { NS }\end{array}$} & \multirow{2}{*}{$\begin{array}{l}0.22 \\
\text { NS }\end{array}$} \\
\hline ASA [150 mg], $n(\%)$ & $102(84.3)$ & $47(79.7)$ & $12(66.7)$ & $59(76.6)$ & & \\
\hline Age, mean \pm SD [years] & $75.9 \pm 13$ & $77.6 \pm 18.7$ & $77.1 \pm 12.4$ & $77.5 \pm 17.2$ & $\begin{array}{l}0.12 \\
\text { NS }\end{array}$ & $\begin{array}{l}0.23 \\
\text { NS }\end{array}$ \\
\hline Body weight, mean \pm SD [kg] & $75.9 \pm 13$ & $77.6 \pm 18.7$ & $77.1 \pm 12.4$ & $77.5 \pm 12.2$ & $\begin{array}{l}0.56 \\
\text { NS }\end{array}$ & $\begin{array}{l}0.70 \\
\text { NS }\end{array}$ \\
\hline $\mathrm{BMI}$, mean $\pm \mathrm{SD}\left[\mathrm{kg} / \mathrm{m}^{2}\right]$ & $27.4 \pm 4.4$ & $27.5 \pm 6.1$ & $28.6 \pm 3.8$ & $27.8 \pm 5.6$ & $\begin{array}{l}0.11 \\
\text { NS }\end{array}$ & $\begin{array}{l}0.57 \\
\text { NS }\end{array}$ \\
\hline Heart rate, mean \pm SD $[\mathrm{bpm}]$ & $73.9 \pm 6.7$ & $75.7 \pm 6.4$ & $76.4 \pm 5$ & $75.9 \pm 6.1$ & $\begin{array}{l}0.10 \\
\text { NS }\end{array}$ & 0.03 \\
\hline $\begin{array}{l}\text { Systolic blood pressure, } \\
\text { mean } \pm \mathrm{SD}[\mathrm{mm} \mathrm{Hg}]\end{array}$ & $134.4 \pm 11.1$ & $133.2 \pm 11.2$ & $137.9 \pm 12.5$ & $134.3 \pm 11.7$ & $\begin{array}{l}0.21 \\
\text { NS }\end{array}$ & $\begin{array}{c}0.81 \\
\text { NS }\end{array}$ \\
\hline $\begin{array}{l}\text { Diastolic blood pressure, } \\
\text { mean } \pm \mathrm{SD}[\mathrm{mm} \mathrm{Hg}]\end{array}$ & $76.6 \pm 7$ & $78.7 \pm 5.5$ & $76.7 \pm 9$ & $78.3 \pm 6.5$ & $\begin{array}{l}0.51 \\
\text { NS }\end{array}$ & $\begin{array}{l}0.13 \\
\text { NS }\end{array}$ \\
\hline NIHSS, mean \pm SD [points] & $4.9 \pm 6.2$ & $4.9 \pm 5.9$ & $3.4 \pm 5.3$ & $4.5 \pm 5.7$ & $\begin{array}{l}0.48 \\
\text { NS }\end{array}$ & $\begin{array}{l}0.98 \\
\text { NS }\end{array}$ \\
\hline mRankin, mean \pm SD [points] & $1.8 \pm 1.5$ & $1.9 \pm 1.4$ & $1.6 \pm 1.2$ & $1.8 \pm 1.4$ & $\begin{array}{l}0.66 \\
\text { NS }\end{array}$ & $\begin{array}{l}0.84 \\
\text { NS }\end{array}$ \\
\hline
\end{tabular}

TIA - transient ischaemic attack, BMI - body mass index, ASA - acetylsalicylic acid, NIHSS - National Institutes of Health Stroke Scale. ${ }^{*}$ Bonferroni pairwise comparisons for proportions, Kruskal-Wallis post-hoc test. ${ }^{* *} \chi^{2}$ test, Mann-Whitney $U$ test.

body mass index (BMI), or mean systolic and diastolic blood pressure. The mean heart rate was significantly different between ASA partial responders and ASA non-responders $(p=0.03$ ) (Table IV). No relationship was found between the occurrence of ASA resistance and any of the risk factors found in patients (Table V).

Diuretics were taken more rarely in ASA non-responders, whereas fibrates were taken more rarely in ASA partial responders (none of the ASA non-responders took any agent from this group). Angiotensin II receptor blockers were significantly more often taken by the group of ASA partial responders and ASA non-responders $(p=0.04)$ (Table VI).

A significantly higher mean haematocrit value ( $p=0.03$ ) was found in ASA partial responders and the ASA non-responder group. The mean LDL concentration was significantly higher in ASA non-responders $(p=0.02)$. No relationship was found between the aggregation parameters and the results of the other laboratory tests (Table VII).

\section{Discussion}

In our study we found the occurrence of ASA resistance in over $9 \%$ of patients taking ASA after a cerebrovascular accident. Similar results were obtained by Berrouschot et al. [11], Seok et al. [12] and Bernstein et al. [13]. A few other researchers observed a lower percentage [13-16]. However, there are also reports of a higher percentage of ASA resistance in patients with a history of a cerebrovascular episode, reaching as high as $60 \%[17,18]$. The above-mentioned authors used different laboratory methods of platelet function assessment, which impairs the comparison of the study results. Numerous conducted studies show a correlation between the results of platelet aggregation obtained by the traditional method (optical aggregometry by Born) and by impedance aggregometry using a multiple platelet function analyser [3, 4, 9]. The latter is much quicker, less laborious and does not require special preparation of the blood sample, hence decreasing the risk of the laboratory error. Therefore, it was chosen for the assessment of platelet function in this study. 
Table V. Cardiovascular risk factors present in all the examined patients and various ASA response groups

\begin{tabular}{|c|c|c|c|c|c|c|c|}
\hline Risk factor & $\begin{array}{c}\text { All pa- } \\
\text { tients } \\
(N=198) \\
n(\%)\end{array}$ & $\begin{array}{c}\text { ASA } \\
\text { responders } \\
(\mathrm{R}) \\
(N=121) \\
n(\%)\end{array}$ & $\begin{array}{c}\text { ASA partial } \\
\text { responders } \\
(\mathrm{PR}) \\
(N=59) \\
n(\%)\end{array}$ & $\begin{array}{c}\text { ASA } \\
\text { non-re- } \\
\text { sponders } \\
(\mathrm{NR}) \\
(N=18) \\
n(\%)\end{array}$ & $\begin{array}{c}\text { ASA partial } \\
\text { responders } \\
\text { and non-re- } \\
\text { sponders } \\
\text { (PR }+N R) \\
(N=77) \\
n(\%)\end{array}$ & $\begin{array}{c}\text { Value of } \\
p^{\star *} \\
\text { R vs. NR }\end{array}$ & $\begin{array}{c}\text { Value of } \\
p^{* * *} \\
R \text { vs. PR }+ \\
\text { NR }\end{array}$ \\
\hline $\begin{array}{l}\text { Arterial } \\
\text { hypertension }\end{array}$ & $163(82.3)$ & $104(85.9)$ & $45(76.3)$ & $14(77.8)$ & $59(76.6)$ & $\begin{array}{l}0.97 \\
\text { NS }\end{array}$ & $\begin{array}{l}0.13 \\
\text { NS }\end{array}$ \\
\hline $\begin{array}{l}\text { Coronary heart } \\
\text { disease }\end{array}$ & $59(29.8)$ & $39(32.2)$ & $14(23.7)$ & $6(33.3)$ & $20(26)$ & $\begin{array}{l}0.99 \\
\text { NS }\end{array}$ & $\begin{array}{l}0.07 \\
\text { NS }\end{array}$ \\
\hline $\begin{array}{l}\text { Previous } \\
\text { myocardial infarct }\end{array}$ & $24(12.1)$ & $15(12.4)$ & $6(10.2)$ & $3(16.7)$ & $9(11.7)$ & $\begin{array}{l}0.46 \\
\text { NS }\end{array}$ & $\begin{array}{l}0.76 \\
\text { NS }\end{array}$ \\
\hline Diabetes mellitus & $60(30.3)$ & $36(29.7)$ & $18(30.5)$ & $6(33.3)$ & $24(31.2)$ & $\begin{array}{l}0.92 \\
\text { NS }\end{array}$ & $\begin{array}{l}0.60 \\
\text { NS }\end{array}$ \\
\hline Dyslipidaemia & $145(73.2)$ & $91(75.2)$ & $38(64.4)$ & $16(88.9)$ & $54(70.1)$ & $\begin{array}{l}0.97 \\
\text { NS }\end{array}$ & $\begin{array}{l}0.70 \\
\text { NS }\end{array}$ \\
\hline Atrial fibrillation & $30(15.2)$ & $21(17.4)$ & $9(15.3)$ & 0 & $9(11.7)$ & $\begin{array}{l}0.35 \\
\text { NS }\end{array}$ & $\begin{array}{l}0.28 \\
\text { NS }\end{array}$ \\
\hline Current smoking & $47(23.7)$ & $27(22.3)$ & $16(27.1)$ & $4(22.2)$ & $20(26)$ & $\begin{array}{l}0.85 \\
\text { NS }\end{array}$ & $\begin{array}{l}0.47 \\
\text { NS }\end{array}$ \\
\hline $\begin{array}{l}\text { Overweight or } \\
\text { obesity* }\end{array}$ & $111(56.1)$ & $68(56.2)$ & $29(49.2)$ & $14(77.8)$ & $43(55.8)$ & $\begin{array}{l}0.42 \\
\text { NS }\end{array}$ & $\begin{array}{l}0.58^{\#} \\
\text { NS }\end{array}$ \\
\hline
\end{tabular}

${ }^{*} B M I>25 \mathrm{~kg} / \mathrm{m}^{2}$, ${ }^{* *}$ Bonferroni pairwise comparisons for proportions, ${ }^{* \star *} \chi^{2}$ test, ${ }^{*} \mathrm{~V}^{2}$ test.

Table VI. Additional medications used in all the examined patients and various ASA response groups

\begin{tabular}{|c|c|c|c|c|c|c|c|}
\hline Medication & $\begin{array}{c}\text { All pa- } \\
\text { tients } \\
(N=198) \\
n(\%)\end{array}$ & $\begin{array}{c}\text { ASA } \\
\text { responders } \\
(\mathrm{R}) \\
(N=121) \\
n(\%)\end{array}$ & $\begin{array}{c}\text { ASA partial } \\
\text { responders } \\
(\mathrm{PR}) \\
(N=59) \\
n(\%)\end{array}$ & $\begin{array}{c}\text { ASA } \\
\text { non-re- } \\
\text { sponders } \\
(\mathrm{NR}) \\
(N=18) \\
n(\%)\end{array}$ & $\begin{array}{c}\text { ASA partial } \\
\text { responders } \\
\text { and non-re- } \\
\text { sponders } \\
(\mathrm{PR}+\mathrm{NR}) \\
(N=77) \\
n(\%)\end{array}$ & $\begin{array}{c}\text { Value of } \\
p^{*} \\
\text { R vs. NR }\end{array}$ & $\begin{array}{c}\text { Value of } \\
p^{\star *} \\
R \text { vs. } \\
P R+N R\end{array}$ \\
\hline Diuretic & $66(33.3)$ & $42(34.7)$ & $22(37.3)$ & $2(11.1)$ & $24(31.2)$ & $\begin{array}{l}0.25 \\
\text { NS }\end{array}$ & $\begin{array}{l}0.6 \\
\text { NS }\end{array}$ \\
\hline ACE inhibitor & $116(58.6)$ & $74(61.2)$ & $33(55.9)$ & $9(50)$ & $42(54.6)$ & $\begin{array}{l}0.37 \\
\text { NS }\end{array}$ & $\begin{array}{l}0.36 \\
\text { NS }\end{array}$ \\
\hline $\begin{array}{l}\text { Angiotensin } \\
\text { II receptor } \\
\text { antagonist }\end{array}$ & $16(8)$ & $6(5)$ & $9(15.3)$ & $1(5.6)$ & $10(13.0)$ & $\begin{array}{l}0.63 \\
\text { NS }\end{array}$ & $0.04 \#$ \\
\hline $\begin{array}{l}\text { Calcium } \\
\text { antagonist }\end{array}$ & $45(22.7)$ & $29(24.0)$ & $10(16.9)$ & $6(33.3)$ & $16(20.8)$ & $\begin{array}{l}0.59 \\
\text { NS }\end{array}$ & $\begin{array}{l}0.30 \\
N S\end{array}$ \\
\hline$\beta$-Blocker & $78(39.4)$ & $49(40.5)$ & $23(40)$ & $6(33.3)$ & $29(37.7)$ & $\begin{array}{l}0.56 \\
\text { NS }\end{array}$ & $\begin{array}{l}0.69 \\
\text { NS }\end{array}$ \\
\hline Nitrate & $31(15.6)$ & $15(12.4)$ & $12(20.3)$ & $4(22.2)$ & $16(21)$ & $\begin{array}{l}0.95 \\
\text { NS }\end{array}$ & $\begin{array}{l}0.11 \\
\text { NS }\end{array}$ \\
\hline Statin & $132(66.7)$ & $84(70)$ & $35(59.3)$ & $13(72.2)$ & $48(62.3)$ & $\begin{array}{c}0.93 \\
\text { NS }\end{array}$ & $\begin{array}{c}0.32 \\
\text { NS }\end{array}$ \\
\hline Fibrate & $7(3.5)$ & $6(5.0)$ & $1(1.7)$ & 0 & $1(1.3)$ & $\begin{array}{l}0.50 \\
\text { NS }\end{array}$ & $\begin{array}{l}0.35^{\# \#} \\
\text { NS }\end{array}$ \\
\hline $\begin{array}{l}\text { Proton-pump } \\
\text { inhibitor }\end{array}$ & $30(15.2)$ & $22(18.2)$ & $4(6.8)$ & $4(22.2)$ & $8(10.4)$ & $\begin{array}{l}0.99 \\
\text { NS }\end{array}$ & $\begin{array}{l}0.13 \\
\text { NS }\end{array}$ \\
\hline
\end{tabular}

ACE inhibitor - angiotensin converting enzyme inhibitor. ${ }^{*}$ Bonferroni pairwise comparisons for proportions. ${ }^{* *} \chi^{2}$ test, ${ }^{\#} V^{2}$ test, ${ }^{\# \#} Y a t e s$ test.

Literature data concerning the relationship between ASA resistance and ASA dose are ambiguous. Some authors have reported a more frequent occurrence of ASA resistance in patients taking smaller doses of ASA $[19,20]$. However, no convincing evidence for the existence of such a correlation was found in large randomized trials [21]. Our study did not demonstrate a relationship be- 
Beata Łabuz-Roszak, Krystyna Pierzchała, Ewa Niewiadomska, Michał Skrzypek, Agnieszka Machowska-Majchrzak

Table VII. Laboratory results (mean \pm SD) in all the examined patients and various ASA response groups

\begin{tabular}{|c|c|c|c|c|c|c|c|}
\hline Test & $\begin{array}{l}\text { All pa- } \\
\text { tients } \\
(N=198)\end{array}$ & $\begin{array}{c}\text { ASA re- } \\
\text { sponders } \\
(\mathrm{R}) \\
(N=121)\end{array}$ & $\begin{array}{c}\text { ASA partial } \\
\text { responders } \\
(\mathrm{PR}) \\
(N=59)\end{array}$ & $\begin{array}{c}\text { ASA } \\
\text { non-re- } \\
\text { sponders } \\
(\mathrm{NR}) \\
(N=18)\end{array}$ & $\begin{array}{l}\text { ASA partial } \\
\text { responders } \\
\text { and non-re- } \\
\text { sponders } \\
(\mathrm{PR}+\mathrm{NR}) \\
(N=77)\end{array}$ & $\begin{array}{l}\text { Value of } p^{*} \\
\text { R vs. NR }\end{array}$ & $\begin{array}{c}\text { Value of } \\
p^{\star *} \\
R \text { vs. } \\
\text { PR + NR }\end{array}$ \\
\hline $\begin{array}{l}\text { Erythrocytes } \\
{\left[10^{6} / \mu \mathrm{l}\right]}\end{array}$ & $4.6 \pm 0.6$ & $4.5 \pm 0.6$ & $4.7 \pm 0.5$ & $4.5 \pm 0.7$ & $4.7 \pm 0.5$ & $\begin{array}{l}0.99 \\
\text { NS }\end{array}$ & $\begin{array}{l}0.13 \\
\text { NS }\end{array}$ \\
\hline Haematocrit [\%] & $40.0 \pm 5.4$ & $39.6 \pm 4.5$ & $40.9 \pm 7$ & $39.9 \pm 5.1$ & $40.7 \pm 6.7$ & $\begin{array}{l}0.88 \\
\text { NS }\end{array}$ & 0.03 \\
\hline $\begin{array}{l}\text { Haemoglobin } \\
{[\mathrm{g} / \mathrm{dl}]}\end{array}$ & $13.8 \pm 1.6$ & $13.7 \pm 0.6$ & $14.2 \pm 1.5$ & $13.3 \pm 2.5$ & $14 \pm 1.8$ & $\begin{array}{l}0.40^{\#} \\
\text { NS }\end{array}$ & $\begin{array}{l}0.06 \\
\text { NS }\end{array}$ \\
\hline $\begin{array}{l}\text { Leukocytes } \\
{\left[10^{3} / \mu \mathrm{l}\right]}\end{array}$ & $8.7 \pm 7.1$ & $9 \pm 8.9$ & $8.1 \pm 2.7$ & $8.7 \pm 2.9$ & $8.3 \pm 2.7$ & $\begin{array}{l}0.57 \\
\text { NS }\end{array}$ & $\begin{array}{l}0.77 \\
\text { NS }\end{array}$ \\
\hline Platelets $\left[10^{3} / \mu \mathrm{l}\right]$ & $244.6 \pm 78.3$ & $242 \pm 73.4$ & $245.6 \pm 84$ & $259.9 \pm 94.9$ & $248.9 \pm 86$ & $\begin{array}{l}0.90 \\
\text { NS }\end{array}$ & $\begin{array}{l}0.66 \\
\text { NS }\end{array}$ \\
\hline APTT [s] & $29.2 \pm 3.7$ & $29.2 \pm 3.7$ & $29.5 \pm 3.8$ & $28.5 \pm 3$ & $29.2 \pm 3.6$ & $\begin{array}{l}0.83 \\
\text { NS }\end{array}$ & $\begin{array}{l}0.85 \\
\text { NS }\end{array}$ \\
\hline INR & $1.1 \pm 0.2$ & $1 \pm 0.1$ & $1.2 \pm 0.3$ & $1 \pm 0.1$ & $1.1 \pm 0.3$ & $\begin{array}{c}0.77 \\
\text { NS }\end{array}$ & $\begin{array}{l}0.25 \\
\text { NS }\end{array}$ \\
\hline $\begin{array}{l}\text { Total cholesterol } \\
{[\mathrm{mmol} / \mathrm{l}]}\end{array}$ & $5.2 \pm 1.2$ & $5.2 \pm 1.2$ & $5 \pm 0.9$ & $5.5 \pm 1.7$ & $5.1 \pm 1.2$ & $\begin{array}{l}0.18 \\
\text { NS }\end{array}$ & $\begin{array}{l}0.93 \\
\text { NS }\end{array}$ \\
\hline LDL [mmol/li] & $3.2 \pm 1$ & $3.2 \pm 1$ & $2.9 \pm 0.9$ & $3.8 \pm 0.9$ & $3.2 \pm 1$ & 0.02 & $\begin{array}{l}0.99^{\# \#} \\
\text { NS }\end{array}$ \\
\hline $\mathrm{HDL}[\mathrm{mmol} / \mathrm{I}]$ & $4.1 \pm 2.5$ & $1.4 \pm 0.5$ & $1.5 \pm 0.5$ & $1.3 \pm 0.3$ & $1.4 \pm 0.4$ & $\begin{array}{l}0.75 \\
\text { NS }\end{array}$ & $\begin{array}{l}0.49 \\
\text { NS }\end{array}$ \\
\hline $\begin{array}{l}\text { Triglycerides } \\
{[\mathrm{mmol} / \mathrm{l}]}\end{array}$ & $1.4 \pm 1.0$ & $1.4 \pm 0.9$ & $1.5 \pm 0.4$ & $1.5 \pm 0.9$ & $1.5 \pm 1.3$ & $\begin{array}{l}0.87 \\
\text { NS }\end{array}$ & $\begin{array}{l}0.88 \\
\text { NS }\end{array}$ \\
\hline $\begin{array}{l}\text { Fasting glucose } \\
{[\mathrm{mg} / \mathrm{dl}]}\end{array}$ & $124.2 \pm 51.1$ & $125.9 \pm 49.3$ & $123.8 \pm 60$ & $113.5 \pm 29.2$ & $121.3 \pm 54.3$ & $\begin{array}{l}0.39 \\
\text { NS }\end{array}$ & $\begin{array}{l}0.45 \\
\text { NS }\end{array}$ \\
\hline $\mathrm{HbA}_{1 \mathrm{c}}[\%]$ & $6.3 \pm 1.3$ & $6.2 \pm 0.8$ & $6.5 \pm 2.1$ & $6.1 \pm 0.9$ & $6.4 \pm 1.9$ & $\begin{array}{l}0.66 \\
\text { NS }\end{array}$ & $\begin{array}{l}0.55 \\
\text { NS }\end{array}$ \\
\hline $\mathrm{CRP}[\mathrm{mg} / \mathrm{l}]$ & $14.1 \pm 21.7$ & $13.4 \pm 21.2$ & $16.6 \pm 25$ & $10.8 \pm 12.1$ & $15.3 \pm 22.7$ & $\begin{array}{l}0.46 \\
\text { NS }\end{array}$ & $\begin{array}{l}0.63 \\
\text { NS }\end{array}$ \\
\hline ALT [U/I] & $24.3 \pm 19.4$ & $23.9 \pm 17.8$ & $22.5 \pm 15.4$ & $21.9 \pm 9.5$ & $22.4 \pm 14.3$ & $\begin{array}{c}0.67 \\
\text { NS }\end{array}$ & $\begin{array}{l}0.82 \\
\text { NS }\end{array}$ \\
\hline AST [U/I] & $27.3 \pm 15.8$ & $29 \pm 22.4$ & $28.5 \pm 17.6$ & $22.7 \pm 5.8$ & $27.3 \pm 16$ & $\begin{array}{l}0.81 \\
\text { NS }\end{array}$ & $\begin{array}{l}0.98 \\
\text { NS }\end{array}$ \\
\hline Bilirubin [mg/l] & $12.6 \pm 5.6$ & $12.3 \pm 5.4$ & $12.8 \pm 5.8$ & $13.1 \pm 7.4$ & $12.9 \pm 6$ & $\begin{array}{l}0.81 \\
\text { NS }\end{array}$ & $\begin{array}{l}0.79 \\
\text { NS }\end{array}$ \\
\hline Creatinine $[\mathrm{mg} / \mathrm{l}]$ & $83.1 \pm 31.1$ & $84.8 \pm 30$ & $83.4 \pm 36.2$ & $68.1 \pm 15.6$ & $79.8 \pm 33$ & $\begin{array}{l}0.24 \\
\text { NS }\end{array}$ & $\begin{array}{l}0.23 \\
\text { NS }\end{array}$ \\
\hline
\end{tabular}

$A P T T$ - activated partial thromboplastin time, INR - international normalized ratio, $L D L$ - low-density lipoproteins, CRP-C-reactive protein, $H b A_{1 c}$ - glycated haemoglobin $A_{11}, H D L$ - high-density lipoproteins, ALT - aspartate aminotransferase, AST - alanine aminotransferase. ${ }^{\star}$ Kruskal-Wallis post-hoc test, ${ }^{* *}$ Mann-Whitney $U$ test, " post-hoc LSD test, ${ }^{\# \# T ~ t e s t . ~}$

tween ASA dose and the resistance phenomenon either.

Similarly to the majority of researchers, we did not observe a relationship between ASA resistance and type of ischaemic stroke, neurological status and functional status of patients [11, 12]. Only Englyst et al. reported the more frequent occurrence of resistance in patients with diagnosed lacunar stroke and in patients with a more severe course of the disease [22].
We found no correlation between platelet function and the gender of patients. Similar reports were presented by Berrouschot et al. [11] and Zytkiewicz et al. [14]. In a few reports ASA resistance was more frequently found in female patients $[3,23]$.

We did not observe a correlation between ASA resistance and age, similarly to the results of some other authors [11, 14]. However, this contrasts with the observations of other researchers, since in their studies the elderly more often showed the 
lack of an anti-aggregatory response to ASA and required the administration of higher doses to achieve this effect $[12,23,24]$.

We did not find a relationship between abnormal platelet function and overweight or obesity, similarly to Zytkiewicz et al. [14]. Some researchers have observed excessive platelet activity and decreased sensitivity to ASA in obese patients, especially those diagnosed with the metabolic syndrome [16, 25].

We found a relationship between LDL cholesterol concentration and the occurrence of ASA resistance, which is also consistent with the medical literature. Friend et al. observed in $69 \%$ of patients with hyperlipidaemia an ineffective platelet response to ASA [26]. Karepov et al. noted a relationship between the lack of anti-aggregation and triglyceridaemia despite ASA intake [16].

The results of some experimental studies show the significant influence of hyperglycaemia on platelet function since hyperglycaemia results in increased prothrombotic condition and proinflammatory functions promoting atherosclerosis and acute vascular episodes by the induction of platelet activation and expression of tissue factor in monocytes. Hyperglycaemia can also be related to decreased sensitivity to ASA [12, 27]. In our study, we did not confirm the reports of other authors concerning the influence of carbohydrate disorders on platelet function in patients who take ASA [28].

Similarly to Macchi et al. [29], we did not confirm the relationship between ASA resistance and smoking, which was reported by some authors [3].

We observed that angiotensin II receptor blockers were significantly more often taken by patients with decreased sensitivity and resistance to ASA. In the available literature such a relationship has not been reported before. Also, in this study platelet aggregation was better in patients taking fibrates, but the group of patients treated with fibrates was small $(n=7)$. In the available literature there are no reports regarding interactions between ASA and fibrates. There are, however, reports concerning the influence of statins on platelet function. Statins reduce the probability of the occurrence of ASA resistance, which may be related to the reduction in cholesterol level [30]. Some authors also reported a relationship between the effects of antiplatelet therapy and the intake of non-steroid anti-inflammatory drugs (NSAIDs) (mainly ibuprofen), calcium channel blockers, selective serotonin reuptake inhibitors, proton-pump inhibitors, ACE inhibitors and $\beta$-blockers [12, 31-36]. Such relationships were not confirmed in our study.

The relationship between the anti-aggregatory ASA effect and the time of ASA intake was also reported in the literature. The majority of researchers noted a higher percentage of ASA resistant patients in the group of patients taking this agent for a long time, which is consistent with our study results $[13,37]$.

In all patients blood was collected in the early hours in the morning. Some authors reported that at this time ASA resistance is more frequent compared to the rest of the day. Excessive platelet activation can be related to catecholamine release in the morning [38]. This phenomenon indirectly explains the relationship between the lack of anti-aggregatory ASA action and higher heart rate, which was observed in this study and by a few other researchers [15].

The relationship between ASA resistance and high haematocrit, which was observed in our study, is also consistent with the data in the literature [39].

As the literature data indicate, patient non-compliance is an important factor which limits the effect of ASA [40]. This factor was eliminated in the group of patients in the acute phase of ischaemic stroke because all patients took ASA during their hospitalization under the control of the nurse. In the group of patients treated chronically, the regularity of ASA intake was determined on the basis of the carefully obtained medical history.

In conclusion, the applied method resulted in detecting the phenomenon of ASA resistance in some patients with TIA and ischaemic stroke (both in acute and chronic phases). The study revealed some possible risk factors of ASA resistance: longer duration of ASA therapy, increased heart rate, higher LDL concentration, and higher haematocrit value. The relationship between resistance to ASA and the intake of some medications (such as angiotensin II receptor blockers, diuretics, fibrates) requires further study. Platelet function monitoring should be considered in patients at a greater risk of ASA resistance.

\section{Conflict of interest}

The authors declare no conflict of interest.

\section{References}

1. Jack DB. One hundred years of aspirin. Lancet 1997; 350: 437-9.

2. Antithrombotic Trialist' Collaboration. Collaborative meta-analysis of randomized trials of antiplatelet therapy for prevention of death, myocardial infarction, and stroke in high risk patients. BMJ 2002; 324: 71-86.

3. Gum PA, Kottke-Marchand K, Poggio ED, et al. Profile and prevalence of aspirin resistance in patients with cardiovascular disease. Am J Cardiol 2001; 88: 230-5.

4. Krasopoulos G, Brister SJ, Beattie WS, Buchanan MR. Aspirin resistance and risk of cardiovascular morbidity: systematic review and meta-analysis. BMJ 2008; 336: 195-8.

5. Feher G, Feher A, Pusch G, et al. Clinical importance of aspirin and clopidogrel resistance. World J Cardiol 2010; 2: 171-186. 
6. Snoep JD, Hovens MC, Eikenboom JC, et al. Association of laboratory-defined aspirin resistance with a higher risk of recurrent cardiovascular events. Arch Intern Med 2007; 167: 1593-9.

7. Fitzgerald R, Pirmohamed M. Aspirin resistance: effect of clinical, biochemical and genetic factors. Pharmacol Therap 2011; 130: 213-25.

8. Toth O, Calatzis A, Penz S, et al. Multiple electrode aggregometry: a new device to measure platelet aggregation in whole blood. Thromb Haemost 2006; 96: 781-8.

9. Paniccia R, Antonucci E, Maggini N, et al. Assessment of platelet function on whole blood by multiplate electrode platelet aggregometry in high-risk patients with coronary artery disease receiving antiplatelet therapy. Am J Clin Pathol 2009; 131: 834-42.

10. Calatzis A, Spannagl M, Loreth R. Multiplate platelet function analysis - application and interpretation. V2.0/07.2007. Dynabyte Medical, Monachium 2007.

11. Berrouschot J, Schwetlick B, von Twickel G, et al. Aspirin resistance in secondary stroke prevention. Acta Neurol Scand 2006; 113: 31-5.

12. Seok JI, Joo IS, Yoon JH, et al. Can aspirin resistance be clinically predicted in stroke patients? Clin Neurol Neurosurg 2008; 110: 110-6.

13. Bernstein PL, Jacobson BF, Connor MD, Becker PJ. Aspirin resistance in south African Caucasian patients with thrombotic cerebrovascular events. J Neurol Sci 2009; 277: 80-2.

14. Zytkiewicz M, Gielwanowska L, Wojtasinska E, et al. Resistance to acetylsalicylic acid in patients after ischaemic stroke. Pol Arch Med Wewn 2008; 118: 727-32.

15. Postula M, Tarchalska-Krynska B, Filipiak KJ, et al. Factors responsible for aspirin resistance - can we identify them? Kardiol Pol 2010; 68: 403-11.

16. Karepov V, Tolpina G, Kuliczkowski W, Serebruany V. Plasma triglycerides as predictors of platelet responsiveness to aspirin in patients after first ischaemic stroke. Cerebrovasc Dis 2008; 26: 272-6.

17. Bennet D, Yan B, Macgregor L, et al. A pilot study of resistance to aspirin in stroke patients. J Clin Neurosci 2008; 15: 1204-9.

18. Zimmermann N, Hohlfeld T. Clinical implications of aspirin resistance. Thromb Haemost 2008; 100: 379-90.

19. Awtry EH, Loscalza J. Aspirin. Circulation 2000; 101: 1026-18.

20. Schror K. Aspirin and platelets: the antiplatelet action of aspirin and its role in thrombosis treatment and prophylaxis. Semin Thromb Hemost 1997; 23: 349-56.

21. Dutch TIA Trial Study Group. A comparison of two doses of aspirin (30 mg vs $283 \mathrm{mg}$ a day) in patients after a transient ischaemic attack of minor ischaemic stroke. N Engl J Med 1991; 325: 1261-6.

22. Englyst NA, Horsfield G, Kwans J, Byrne CD. Aspirin resistance is more common in lacunar strokes than embolic strokes and is related to stroke severity. J Cereb Blood Flow Metab 2008; 28: 1196-203.

23. Macchi L, Christaens L, Brizard A, et al. Resistance to aspirin in vitro is associated with increased platelet sensitivity to adenosine diphosphate. Thromb Res 2002; 107: 5-49.

24. Alberts MJ, Bergman DL, Molner E, et al. Antiplatelet effect of aspirin in patients with cerebrovascular disease. Stroke 2004; 35: 175-8.

25. Santilli F, Vazzana N, Liani R, et al. Platelet activation in obesity and metabolic syndrome. Obes Res 2012; 13: 27-42.
26. Friend M, Vucenik I, Miller M. Platelet responsiveness to aspirin in patients with hyperlipidemia. BMJ 2003; 326: 82-3.

27. Vaidyula VR, Boden G, Rao AK. Platelet and monocyte activation by hyperglycemia and hiperinsulinemia in healthy subjects. Platelets 2006; 17: 577-85.

28. DiChiara J, Bliden KP, Tantry US, et al. The effect of aspirin dosing on platelet function in diabetic and nondiabetic patients an analysis from the aspirin-induced platelet effect (ASPECT) Study. Diabetes 2007; 56: 3014-9.

29. Macchi L, Christiaens L, Brabant S, et al. Resistance in vitro to low dose aspirin is associated with platelet PIA1 (GPIIIa) polymorphism but not with C807T (GPIa-IIa) and C-5T Kozak (GP Ibalpha) polymorphisms. J Am Coll Cardiol 2003; 42: 1115-9.

30. Neubauer H, Kaiser AF, Endres HG, et al. Tailored antiplatelet therapy can overcome clopidogrel and aspirin resistance: the BOchum CLopidogrel and Aspirin Plan (BOCLA-Plan) to improve antiplatelet therapy. BMC Med 2011; 9: 3.

31. Tirnaksiz E, Pamukcu B, Oflaz H, Nisanci Y. Effect of high dose statin therapy on platelet function; statins reduce aspirin resistant platelet aggregation in patients with coronary heart disease. J Thromb Thrombolysis 2009; 27: 24-8.

32. Siller-Matula JM, Spiel AO, Lang IM, et al. Effects of pantoprazole and esomeprazole on platelet inhibition by clopidogrel. Am Heart J 2009; 157: 148-53.

33. Canella-Lawson F, Reilly MP, Kapoor SC, et al. Cyclooxygenase inhibitors and the antiplatelet effects of aspirin. N Engl J Med 2001; 345: 1809-17.

34. Kurth T, Glynn RJ, Walker AM, et al. Inhibition of clinical benefits of aspirin on first myocardial infarction by nonsteroidal anti-inflamatory drugs. Circulation 2003; 108: 1191-5

35. Hergovich N, Aigner M, Eichler HG, et al. Paroxetine decreases platelet serotonin storage and platelet function in human beings. Clin Pharmacol Ther 2000; 68: 435-42.

36. Siller-Matula JM, Lang IM, Christ G, Jilma B. Calcium-channel blockers reduce the antiplatelet effect of clopidogrel. J Am Coll Cardiol 2008; 52: 1557-63.

37. Pulcinelli FM, Pignatelli P, Celestini A, et al. Inhibition of platelet aggregation by aspirin progressively decreases in long-term treated patients. J Am Coll Cardiol 2004; 43: 979-84

38. Velasco A, Coma-Canella I, Moreno J. Aspirin resistance has a circadian rhythm. J Coronary Artery Dis 2005; 6: 67-8.

39. Roller RE, Dorr A, Ulrich S, Pilger E. Effect of aspirin treatment in patients with peripheral arterial disease monitored with the platelet function analyzer PFA-100. Blood Coagul Fibrynolysis 2002; 13: 277-81.

40. Kenneth A, Schwartz L, Schwartz DE, et al. Non-compliance is the predominant cause of aspirin resistance in chronic coronary arterial disease patients. J Trans Med 2008; 6: 46-52. 